\title{
Stochastic and continuous gravitational wave analysis pipelines for pulsar timing array data
}

\author{
Justin Ellis ${ }^{1}$, Fredrick Jenet ${ }^{2}$, Xavier Siemens ${ }^{3}$ \\ and Maura McLaughlin ${ }^{4}$ \\ ${ }^{1}$ University of Wisconsin Milwaukee, United States \\ email: justin.ellis18@gmail.com \\ ${ }^{2}$ University of Texas, Brownsville \\ email: fredrickajenet@gmail.com \\ ${ }^{3}$ University of Wisconsin, Milwaukee \\ email: siemens@gravity.phys.uwm.edu \\ ${ }^{4}$ West Virginia University \\ email: maura.mclaughlin@mail.wvu.edu
}

\begin{abstract}
The Nanohertz Observatory for Gravitational Waves (NANOGrav) collaboration aims to detect gravitational waves (GWs) through the precise timing of millisecond pulsars. GWs will come in the form of a stochastic background, continuous sources and burst sources. Here we will review recent progress on the development of data analysis pipelines aimed at the detection of a stochastic background as well as continuous sources. We will introduce the Optimal Statistic and F-Statistic methods that are used in the stochastic and continuous pipelines, respectively. Both pipelines are fully functional on real pulsar timing data and take into account the timing models for each pulsar. Finally, we will present the efficacy of each pipeline on locally simulated data as well as data from the 2012 IPTA data challenge.
\end{abstract}

